\title{
El turismo urbano como oferta turística alternativa en Medellín: comportamientos espaciales de la ciudad como destino turístico
}

\section{Urban tourism as alternative tourism in Medellin: spatial behaviors of the city as a tourist destination}

\author{
Hernando Bejarano Arismendi"; María E. Arroyave Torres**; Martha E. Saldarriaga Ospina ${ }^{* * *}$; \\ Carlos A. Urrego Ramírez ${ }^{* * * *}$ Diego A. González Muñoz ${ }^{* * * * *}$ \\ herbeja@yahoo.com; maria.arroyave@usbmed.edu.co; martha.saldarriaga@usbmed.edu.co; \\ carlosurregor@gmail.com; diego.gonzalez2586@gmail.com
}

Recibido: marzo 5 de 2017. Aceptado: mayo 31 de 2017.

doi: 10.22209/rhs.v5n1a02

\section{Resumen}

El proyecto de investigación «Actualización e implementación del inventario de atractivos turísticos de la ciudad de Medellín y sus corregimientos», en convenio de asociación con la Subsecretaría de Turismo de la Alcaldía de Medellín realizado para el año 2016, dejó los siguientes interrogantes: ¿Existe una oferta clara de recorridos turísticos, tanto culturales como ambientales, en Medellín?, y ¿cómo pueden los recursos identificados y valorados en el inventario turístico contribuir a que dicha oferta satisfaga las necesidades del turista que llega a Medellín actualmente? Abordar este cuestionamiento es fundamental en este artículo, que busca una reflexión acerca del reconocimiento y valoración de la oferta turística local y el modelo de gestión de la ciudad desde sus inicios. Esta reflexión permite evidenciar manifestaciones y comportamientos espaciales que generan hoy oportunidades a las comunidades de una manera incluyente e irradian una nueva imagen de ciudad emergente, resiliente de sus dificultades e innovadora en sus acciones, como pocas en el mundo; también permite identificar nuevas oportunidades de ciudad para su ordenación e intervención planificadora hacia el futuro, en las que todos deben prepararse para aumentar y conservar la posibilidad de optimizar el desarrollo económico y social local de manera sostenible y competitiva.

Palabras clave: transformación, resiliencia, turismo cultural urbano y rural, espacialidad turística local, ordenación y planificación territorial turística.

* Arquitecto por la Universidad Nacional de Colombia; especialista en Planificación U. . lombia; especialista en Gerencia por la Institución Universitaria ceipa; magíster en Dirección de Empresas por la Escuela de Administración de Empresas de Barcelona, España y especialista en Gerencia de Entidades Sociales con énfasis en Desarrollo Local y Marketing Territorial por la Universidad EAfit. Asesor y Consultor en proyectos turísticos.

** Magíster en Administración de Empresas con especialidad en Dirección de Proyectos por la Universidad Viña del Mar, Chile; especialista en Gerencia de Servicios Sociales por la Fundación Universitaria Luis Amigó e Ingeniera de Sistemas por la Universidad Cooperativa de Colombia. Directora del Programa de Administración Turística y coordinadora del Semillero de Investigación Innovación y Empresas Sostenibles - Universidad de San Buenaventura. Asesora y consultora en proyectos del sector público y privado.

*** Administradora de Empresas Turísticas por la Institución Universitaria Colegio Mayor de Antioquia; licenciada en Administración Educativa por la Universidad de San Buenaventura; magíster en Administración por la Universidad EAfiт. 33 años de experiencia docente de cátedra en pregrado y postgrado. Consultora certificada en Certificación de Competencias de Consultoría. Asesora y consultora en proyectos turísticos.

**** Comunicador Social por la Universidad de Antioquia; técnico en Hotelería y Turismo por el Instituto Colombo Europeo. Conferencista en la Institución Universitaria Colegio Mayor de Antioquia, EsUMER, Corporación Turística del Suroeste y Universidad de San Buenaventura. Asesor y consultor en proyectos turísticos.

***** Administrador de empresas turísticas por la Institución Universitaria Colegio Mayor de Antioquia. Docente universitario de cátedra en pregrado. Asesor y consultor en planificación y gestión de destinos. 


\title{
RHS Revisa Humansmo RHS ysocectad
}

\section{Artículo de reflexión}

\begin{abstract}
The research project "Update and implementation of the inventory of Medellín city's tourist attractions and its districts", carried out in 2016 in collaboration with the Undersecretariat of Tourism of the City Hall of Medellín, raised the following questions: Is there a clear offer of cultural and environmental tourist tours in Medellín? How can the resources identified and assessed in the tourism inventory contribute to an offer that meets the needs of tourists visiting Medellín today? This paper focuses on these questions and aims to reflect on the recognition and assessment of the local tourism offer as well as on the management model of the city from its inception. This analysis shows manifestations and spatial behaviors that create inclusive opportunities for communities, and project a new image of an emergent city which is resilient in the face of difficulties and innovative in its actions, like few in the world. It also allows to identify new opportunities for urban planning and future intervention, for which everyone must be willing to increase and maintain the possibility of optimizing local economic and social development in a sustainable and competitive way.
\end{abstract}

Keywords: transformation, resilience, cultural urban and rural tourism, local spatial tourism, tourist land regulation and planning.

\section{Introducción}

El turismo presente en la ciudad de Medellín no ha sido una prioridad histórica y su desarrollo es el resultado fortuito de condiciones o respuestas a otras problemáticas no propiamente turísticas, como: la movilidad urbana, la recuperación de espacios públicos urbanos y rurales, dar respuestas a las demandas sociales en educación, los servicios públicos, los escenarios de conocimientos e investigación, las infraestructuras deportivas, los equipamientos al servicio de las comunidades, la protección a las zonas de reservas naturales, la mejora de las condiciones ambientales de ciudad, entre otros. En todas estas condiciones fortuitas, el turismo ha logrado manifestarse y ser relevante a través de mejoras, al diseñarse recorridos turísticos utilizando recursos o bienes que se consolidan en el día a día de manera alternativa, tales son los casos del turismo de innovación social, cultural, rural-urbano, de eventos científicos y sociales, de compras, gastronómico y turismo de identidad cultural, entre otros. En dichas tipologías de turismo los sistemas de transporte, la arquitectura, la preservación patrimonial, los servicios turísticos, las fiestas tradicionales, la regeneración urbana, los espacios de integración, la oferta eco urbana, entre muchos otros, son elementos que atraen la atención de viajeros extranjeros, con un mercado que según la Organización Mundial del Turismo (омт) se estima en cerca de 270 millones de viajeros (o turistas) que demandan productos turísticos auténticos en las urbes turísticas hoy existentes.

De los procesos que la ciudad desde su administración pública ha planteado y ha consolidado, se reconoce el espacio para eventos, ferias y reuniones de mediano formato en el ámbito latinoamericano con una arquitectura en su gobernanza local entre lo público y privado, con el propósito de mitigar el aislamiento que la ciudad por más de una década mantuvo, debido a un oscuro proceso social violento, que marginó a Medellín de los circuitos de viaje del turismo nacional e internacional; ya que irradiaba una imagen de ciudad peligrosa, insegura e inviable. Esta estrategia ha cumplido un rol para un momento histórico y no se puede definir hoy como la única razón de la ciudad para motivar sus flujos de visitantes hacia esta. Por lo 
tanto, se requieren nuevas acciones estratégicas y de prospectiva turística ante una demanda diversa en constante cambio, cada vez más sofisticada y personalizada, con sensibilidad social, ambiental y cultural, y cuya duración de viajes en el turismo urbano es normalmente corta (de uno a tres días); este tipo de turismo asociado a descansos cortos, en ocasiones, está conectado a una oferta especial o a un evento (festivales o conciertos, congresos, entre otros), de ahí los retos y oportunidades para la ciudad.

\section{Necesidades de la ciudad de Medellín como destino turístico emergente}

Según el informe de Procolombia (2016): «Turismo extranjero en Colombia», el incremento de la llegada de turistas internacionales en el año 2016 con relación al año 2015 fue del $21.6 \%$, lo que corresponde a 331139 viajeros, alcanzando un porcentaje de participación del mercado turístico nacional del $12.8 \%$; con base en estas cifras, la ciudad hoy se puede suscribir como destino turístico emergente estando por encima de la media de crecimiento mundial del 3.9\% según la омт. Comprender las implicaciones de ser un destino de clase mundial contiene en sí mismo una serie de retos para la ciudad en cuanto a la generación de oportunidades sociales y económicas. Supone un desafío para la ciudad y para su administración de ir más allá de asimilar la actividad del turismo como un sector con miradas desde lo territorial, al ampliar esquemas mentales convencionales que históricamente han desarrollado los destinos tradicionales de finales del siglo $\mathrm{xx}$, sean estos nacionales o internacionales.

Según Procolombia, tomando lo dicho por Naciones Unidas, la mitad de la población del mundo es urbana y se espera que para el año 2030 tenga un crecimiento del $61 \%$. Esto quiere decir que, para ese año, el volumen de la población viviendo en ciudades incrementará a 5 billones, lo que tendrá un impacto considerable en el turismo urbano como factor clave del desarrollo de la ciudad y su economía (Procolombia, 2016).

Al insertarse la ciudad en esta dinámica de crecimiento poblacional y entendiendo la posición que viene tomando Medellín como destino turístico emergente, de acuerdo con los resultados del proyecto de investigación «Actualización e implementación del inventario de atractivos turísticos de la ciudad de Medellín y sus corregimientos» surgen los siguientes interrogantes: ¿Existe una oferta clara de recorridos turísticos, tanto culturales como ambientales, en Medellín?, y ¿cómo pueden los recursos identificados y valorados en el inventario turístico contribuir a que dicha oferta satisfaga las necesidades del turista que llega a Medellín actualmente? Los turistas eligen la mejor oferta en relación con la calidad-precio; tienen la necesidad de sentirse como «locales» para integrarse a profundidad; buscan conocer además de la ciudad, también destinos aledaños: por esto es importante descentralizar las áreas turísticas, así como la oferta experiencial que implica remirar y reconceptualizar lo que hoy existe como propuesta. Hoy la ciudad de Medellín podría ser considerada un laboratorio turístico en construcción, entendiendo este concepto como un espacio abierto de interacción, imaginación y trabajo colaborativo entre los diferentes actores de la actividad turística local, en el que confluyen iniciativas y matices, que son de interés a flujos sensibles y motivados por lo social, cultural, ambiental, la reconstrucción física de comunidades, de avances tecnológicos; de ahí que las tareas por realizar, por parte de los actores en cuestión, sean consolidar procesos y generar nuevos productos turísticos alternativos para ser considerada un destino de interés para los flujos internacionales.

Tras la realización de la actualización e implementación del inventario de atractivos turísticos de la ciudad de Medellín y sus corregimientos, como ejercicio planificador del territorio, se requiere revisar en detalle aquellos recursos turísticos significativos tanto del orden cultural como natural encontrados a lo largo de la ciudad y sus corregimientos, para una zonificación y definición de tendencias de la oferta y la demanda. Esta revisión permite categorizar y valorar los recursos turísticos locales, realizar una interpretación, priorización, jerarquización de los recursos, para llegar a un proceso de zonificación espacial turística en términos de oportunidades para el turismo local. El uso de estos instrumentos posibilita tomar decisiones estratégicas y prospectivas desde lo espacial y de la actividad en términos de los requerimientos de las demandas emergentes y necesidades de los actores locales o comunidades, así como incrementar la capacidad de gestión no solo territorial, sino promocional y de marketing local.

La ciudad de Medellín no es hoy un destino turístico per se. El turismo debe conceptualizarse como el valor agregado a motores de desarrollo económico, social, ambiental y cultural, lo cual se evidencia en los procesos de desarrollo de la actividad en los últimos años. 
Individualmente, cada recurso identificado y valorado en la actualización del inventario turístico de la ciudad de Medellín y sus corregimientos, por sí solo, no tiene la suficiente fuerza atractiva para generar corriente de viajeros; por ende, se produce en los últimos años en Medellín un ejercicio de vinculación y asociación relativa de recursos y de posibles servicios. Se tiene como muestra, muchas obras de infraestructura o de soporte del territorio que encuentran en el turismo un relanzamiento y empoderamiento que no lo tenía previsto; ejemplo de ello son algunas estaciones del Metro, Metrocables, tranvía, escaleras eléctricas, puentes, espacios públicos, las uva (Unidades de Vida Articulada), edificios institucionales, entre otros.

La dinámica encontrada en la oferta de recursos turísticos de la ciudad de Medellín denota que está hoy incursionando en el turismo urbano y debe verse como laboratorio turístico vivencial y experiencial de la cultura y lo social. En Medellín se reconocen los aportes de las obras de infraestructura de alta calidad en comunidades vulnerables y pobres; la resistencia desde la actividad cultural ante la violencia y el desapego de valores y principios ciudadanos; el rescate de lo rural y una cultura tradicional a través de eventos y ferias; la presencia del Estado en esas comunidades con iniciativas y proyectos, los cuales revalorizan entornos paisajísticos y ambientales de ciudad al servicio de las comunidades propias y de visitantes, como espacios públicos semirrurales y rurales acercando lo rural a lo urbano.

Se considera a Medellín un laboratorio de aprendizaje turístico, porque permite ir más allá de los conceptos tradicionales, nos da pie a interpretar que los recursos turísticos existentes no deben ser sujetos de análisis individualmente, sino en complementariedad y sinergia. Allí podemos encontrar la masa crítica para generar razones de viaje a la ciudad, como su ubicación entre montañas, que tiene un paisaje biodiverso, una sociedad heterogénea, pero con un común denominador en sus raíces y cultura paisa, se identifican con una música, gastronomía, arraigo por la tierra y los suyos (Marchena Gómez y Vera Rebollo, 1997).

Con el ánimo de reinterpretar la ciudad como destino turístico, sus vocaciones y potencialidades, el proyecto de investigación «Actualización e implementación del inventario de atractivos turísticos de la ciudad de Medellín y sus corregimientos», basado en la metodología de levantamiento de inventarios turísticos del
Ministerio de Comercio, Industria y turismo - Viceministerio de Turismo y de acuerdo con el análisis de la oferta de recursos de ciudad agrupados por unidades espaciales turísticas, conceptos aportados por Robert Boullon (1985) en sus estudios sobre el espacio turístico en suelos rurales y urbanos, para el caso de Medellín, se asimilan y validan seis figuras de las propuestas hechas por Boullon (zonas, áreas, conjuntos, unidades, corredores y circuitos); permitiendo potenciar la oferta en función de temas, tipologías, características o propósitos de ciudad, que le pueden dar carácter y razones por las cuales visitar esos sitios de interés de ciudad.

\section{La espacialidad turística local, oportunidades y retos}

La ciudad viene reconociendo recorridos temáticos que dan identidad a porciones del territorio urbano, es el caso de la La ciudad viene reconociendo recorridos temáticos que dan identidad a porciones del territorio urbano, es el caso de la Vía Primavera, calle de la Buena mesa, entre otros. Al reconocer la oferta del territorio, se requiere una intencionalidad para asociar los elementos y recursos existentes que permitan oportunidades en temas culturales, artísticos, creativos, de moda, pintura, deportivos, gastronómicos, innovación social y cultural, los cuales pueden propiciar recorridos de ciudad en clave de sostenibilidad y competitividad territorial. Como ejemplo de ello tenemos recorridos entre las uva (Unidad de Vida Articula) de mayor potencialidad y accesibilidad, recorridos entre parques bibliotecas, centros deportivos, recorridos procesionales, recorridos entre edificios de representatividad arquitectónica (edificios del centro de ciudad), recorridos gastronómicos (Ciudad del Río y Avenida Jardín), recorridos de transformación social, cultural y económica, recorridos por teatros o por museos, entre otros. Todo ello se constituye en los nuevos escenarios para la implementación de oportunidades de servicios y actividades desde la economía de la creatividad o naranja, las cuales apalancan los desarrollos de la economía local y oportunidades de identidad y valorización del territorio.

Otras potencialidades serían la restauración ambiental de los cerros tutelares El Volador y Nutibara, complementadas con el proyecto Parque del Río, el Metrocable hasta el cerro El Picachito, Jardín Circunvalar, recuperación de parques lineales o corredores urbanos bióticos como La Presidenta, La Iguana, Picachita, los 
cuales estarían vinculados a recorridos temáticos paisajísticos y de avistamiento de flora y fauna de ciudad.

Hoy la ciudad presenta una oferta interesante, si se asocian y articulan recursos relevantes como aquellos meramente reconocidos, en un ejercicio de construcción de producto relacionado a la cultura y naturaleza, donde se hace evidente la capacidad de transformar, adaptar, vincular las actividades humanas al territorio en la generación de valor. Allí vamos a tener un motor que ayuda y potencia a la ciudad, con el cual el turismo de reuniones y convenciones, el turismo científico y de servicios médicos, pueden encontrar un aliado potente para la generación de complementariedad.

El turismo de naturaleza complementa y apoya al turismo de ciudad, dada la cercanía a suelos rurales, a entornos ambientales con capacidades instaladas como los corregimientos de Santa Elena (Parque Arví), San Sebastián de Palmitas (granjas agro turísticas), pero también de admiración y valoración de corredores bióticos urbanos (La Iguana, Picachita, La Presidenta) o desde los Metrocables que permiten ver y sentir el paisaje de una ciudad entre montañas, cerros tutelares y de clima primaveral durante los 365 días del año.

Hoy el turismo comunitario es otra alternativa poderosa para el desarrollo de zonas de la ciudad que históricamente han estado marginadas y casi inviables desde lo socioeconómico. Es el caso de las intervenciones y correlaciones entre el desarrollo urbanístico y demandas sociales, como por ejemplo la comuna 13 , las cuales han permitido la creación de nuevas alternativas para el disfrute de actividades directamente ligadas con el turismo, desde lo cultural, ambiental y social con nuevos espacios públicos, corredores bióticos y referentes o hitos urbanos (escaleras eléctricas). En el oriente de la ciudad de Medellín se viene gestando un escenario de turismo comunitario viable (Moravia), gracias a las estructuras ya existentes y a la vinculación directa de la comunidad con ciertas actividades que se pueden alinear con el turismo en el marco de una oferta de servicios más integral y atrayente; es así como surgen nuevas dinámicas sociales, culturales y económicas para las comunidades. Aquí es donde aparecen las uva (Unidades de Vida Articulada) como espacios fácilmente articulables a este posible escenario y como puntos intermedios dentro de una oferta social y cultural más amplia para la ciudad.
El expansionismo urbano segrega rápidamente las posibilidades de desarrollar actividades al aire libre en los pocos sitios que se consideran con bondades, que se encuentra en laderas que sirven de vaso comunicante con la ruralidad del municipio hacia los corregimientos de: Santa Elena, AltaVista, San Cristóbal, San Sebastián de Palmitas y San Antonio de Prado; a través de senderos, caminos de herradura, paisajes boscosos de reserva local y sistemas productivos agrícolas de orden local. Estos son algunos elementos que se conjugan alrededor de nuevas alternativas que la ciudad puede aprovechar en materia turística, como el Jardín Circunvalar, el Parque Arví y el Corredor Turístico de Ayacucho, que aparecen como detonantes para que esta iniciativa de integración, sumada a otros elementos que, desde la vocación del territorio, caso de las flores, hortalizas, frutas, gastronomía local, se tornan emblemáticas en la ciudad.

El turismo en su tarea de revitalización social y económica de los territorios aparece como un impulsor del progreso y desarrollo; aun así, la concentración de las actividades turísticas en un solo sector puede llegar a desgastar tanto a los sitios (recursos o atractivos) como a la misma comunidad que vive el día a día con los movimientos que genera el turismo en su entorno. Es por eso que se propone de alguna manera la descentralización de la oferta congregada en algunos sitios específicos de la ciudad.

A nivel rural, el reto está en dar fuerza a los caminos ancestrales prehispánicos e hispánicos y su articulación con el parque Arví del corregimiento de Santa Elena; recuperar los caminos ancestrales de los corregimientos de San Sebastián de Palmitas, AltaVista, y los recorridos agro turísticos relacionados con la gastronomía local, actividad productiva, en el corregimiento San Cristóbal y San Antonio de Prado, que pueden ser asociados a temas de turismo de bienestar, entre otros.

Otro reto a nivel urbano sería el conjunto administrativo e institucional de ciudad donde están ubicados: Plaza Mayor, el Teatro Metropolitano, el Edificio Inteligente, el Parque de los Pies Descalzos, el Centro Administrativo la Alpujarra, la Plaza de la libertad, entre otros, que permiten recorridos e itinerarios desde la institucionalidad y sus contenidos de gobernanza de lo público. 
En cuanto al centro de la ciudad, la representatividad de los atractivos turísticos del centro son $100 \%$ patrimonio cultural (Basílica Menor Nuestra Señora de la Candelaria, Centro Administrativo La Alpujarra José María Córdova, Museo de Antioquia, entre otros), específicamente del tercer nivel de clasificación, arquitectura religiosa e institucional, de acuerdo con los parámetros establecidos por la metodología de levantamiento de inventarios turísticos del Ministerio de Comercio, Industria y turismo - Viceministerio de Turismo. En el centro tradicional o histórico, como también se conoce, se concentra la gran mayoría de los atractivos turísticos del patrimonio cultural de la ciudad; se concluye que el turismo cultural es un producto turístico relevante conjuntamente con el turismo de reuniones, negocios y convenciones, pero que cuenta con un gran potencial para consolidarse como un producto principal en la oferta de ciudad, al complementarse con el producto rural y ambiental de su centralidad y de los corregimientos.

El 51\% de los atractivos inventariados en el 2016, en el proyecto de investigación realizado en convenio con la Universidad de San Buenaventura y la Subsecretaría de Turismo de la ciudad, corresponde a edificaciones patrimoniales que actualmente están declarados como bienes de interés cultural (Edificios Carré y Vásquez, Casa Natal de Francisco Antonio Zea, Palacio de la Cultura Rafael Uribe Uribe, entre otros); se concluye que, aunque existe buena representatividad, no se consideran como materia prima para el desarrollo de nuevos productos turísticos por la connotación local que representan sus historias y arquitecturas individuales, pero puede ser un tema de interés si se asocian y vinculan a guiones e historias relevantes con una proyección ante el mercado urbano internacional.

Se recomienda priorizar la proximidad de los atractivos ubicados en las áreas turísticas, mediante un pasaje o corredores peatonales y turísticos, teniendo en cuenta la dinámica que concurren cada uno de ellos desde el enfoque del espacio turístico donde se encuentran; lo anterior es con la finalidad de facilitar la movilidad y distribución de las diferentes corrientes turísticas que convergen en el centro.

Se considera un elemento clave para establecer estrategias del producto turístico cultural de la ciudad, la articulación de espacios públicos como las esculturas que han sido donadas por el maestro Fernando Botero, aprovechando el posicionamiento y reconocimiento que tiene uno de los grandes hijos que ha dado esta ciudad, teniendo en cuenta la previa autorización del propietario de la marca Botero.

El mejoramiento de los espacios públicos en las zonas priorizadas, por medio de intervenciones esquematizadas desde el Plan de Desarrollo de Medellín «Cuenta Con Vos» 2016-2019, permitirá a dichos espacios generar herramientas que faciliten la apropiación de los mismos e implementación de una cultura ciudadana, por lo cual se concluye la importancia de establecer estrategias para lograr una transformación asertiva que beneficie al ciudadano y, por ende, a los visitantes y turistas.

No todos los elementos inventariados obedecen a la calidad de atractivo turístico, por lo cual se concluye la importancia de seleccionar aquellos que, por su representación, posicionamiento y reconocimiento, además de proximidad a otros atractivos, se pueden contemplar para conformar la lista actualizada de los atractivos turísticos referentes de ciudad, y será el insumo principal para desarrollar nuevos productos turísticos e innovar los existentes.

Teniendo en cuenta el comportamiento de la demanda y las corrientes turísticas que se dan en la ciudad, y los espacios que denotan la proximidad y realzan algunos atractivos turísticos que confluyen en tales lugares, es importante implementar acciones de mejoramiento ante el deterioro que se evidencia en algunos de ellos, lo cual requiere de una intervención urgente para facilitar la apropiación de los mismos por parte de sus habitantes y visitantes. Es importante realizar señalizaciones turísticas, remozamiento de espacios públicos para solucionar la dificultad que se presenta en la proximidad en algunos de los atractivos, no solo en el área del centro histórico como foco de especial intervención, sino con aquellas zonas vecinas que concentran diversidad de atractivos turísticos, como el caso de la zona Nororiental de la ciudad.

El desarrollo aislado de la Zona Norte (Carabobo) como distrito de innovación debe ser enlazado a la oferta de ciudad. Hoy los recursos y atractivos que se ubican en esta zona o cerca de la misma se consideran de los más importantes en cuanto a reconocimiento y calidad en la ciudad, lo que supone que todos los cambios que se han generado alrededor no son en vano y que, por el contrario, como sitios de interés para la 
ciudad necesitan de otro tipo de esfuerzos institucionales que pongan aún más en valor dicho sector.

El crecimiento acelerado y desordenado del tejido urbano segrega rápidamente las posibilidades de desarrollar actividades al aire libre en pocos sitios, lo cual se evidencia en el porcentaje del espacio público disponible para la ciudad, que no llega a cumplir normas nacionales e internacionales; así, propuestas como El Jardín Circunvalar asociadas al Gran Corredor Turístico de Ayacucho, que aparecen como detonante para esta iniciativa de nuevos espacios de ciudad, agregando otros elementos que desde la vocación de un territorio como el corregimiento de Santa Elena suman a la urbe un conjunto paisajístico agrícola alrededor de la producción de flores, tema emblemático en la ciudad; la localización de la Placita de Flórez a lo largo de este recorrido es un elemento que permite conectar las historias entre la ruralidad y la parte urbana del municipio. La administración local ha planeado desde años atrás sobre esta área la idea de crear una silleta gigante en el techo de la Placita de Flórez que simule y evoque los colores y olores primaverales del campo en la ciudad.

El fortalecimiento de áreas como Vía Provenza y Vía Primavera en el sector del poblado, calles que emulan los mejores epicentros de moda de Europa, necesitan ser tenidas en cuenta como nuevos ejes de desarrollo cultural y económico para la ciudad. Moda, gastronomía y arte convergen en este lugar dando cabida a nuevas actividades como complemento a otros servicios que se encuentran allí.

La conectividad entre las diferentes áreas de esta zona hace posible la diversificación de la oferta turística que se emplaza en esta. El sistema Metro en su conjunto cubre a cabalidad con dicho fin al poner a disposición de la ciudad un sistema completamente articulado que acorta los desplazamientos. El Metro con sus líneas A, K, L1, L2 y T-A, además de todo el sistema de buses alimentadores, son la punta de lanza hacia nuevos recorridos de ciudad que soporten el diseño de productos turísticos de clase mundial para mercados turísticos urbanos. En materia de accesibilidad para personas con movilidad reducida son pocos los lugares que se prestan a la hora de vincular a este segmento de la población, es una tarea de la ciudad hacia la inclusión. Los parques y otros lugares públicos son aquellos espacios que solo de alguna manera se adhieren a la implementación de cintas táctiles u otros elementos adicionales a las rampas y barandales que muchas veces por norma deben de instalarse. En este sentido, la mayoría de los recursos y atractivos turísticos de la zona carecen de este tipo de equipamientos e infraestructuras, aunque es de reconocer el esfuerzo local en la última señalización turística realizada en el centro, donde se incorpora el lenguaje braille; una de las líneas estratégicas a las cuales, desde el Plan de Desarrollo Turístico de Medellín 2011-2016, se apuesta para el desarrollo de productos turísticos claves para el territorio y con especial interés.

El turismo cultural, debido al nuevo mercado que arriba a la ciudad, en especial el mercado vacacional, requiere de una nueva oferta que vincule elementos particulares y propios de la cultura paisa. Es en este punto en el cual se necesita que la articulación de una nueva apuesta de turismo vincule aspectos arraigados en la idiosincrasia y cultura antioqueña, como la devoción mariana y crística, en general religiosa de todo el pueblo antioqueño, los cuales de alguna u otra manera ya poseen un contenido y se prestan para gestar, a partir de estas condiciones, nuevos escenarios o espacios turísticos de interés, detonantes de la consolidación de la ciudad de Medellín como destino.

Existe un elemento en particular en la ciudad que es propicio para dicha lógica, El Santuario de la Madre Laura, sitio que hoy presenta ya un flujo de visitas por parte de turistas cautivados por la historia e importancia de la santa que cobija con su nombre este lugar. Este se enlaza como conjunto religioso con una serie de templos en la ciudad de Medellín que vinculan historia, arquitectura y culto, y se puede constituir en un circuito o recorrido de gran importancia para la ciudad.

La comuna 13, reconocida nacional e internacionalmente por su pasado oscuro, hoy llena de color la zona centro occidental de Medellín. La transformación social como pilar y estilo de vida de la comunidad ha trazado un nuevo futuro para todos los habitantes de este sector de la ciudad.

El turismo en su tarea de revitalización social y económica de los territorios aparece en este sector como un jalonador de progreso y desarrollo; aun así, la concentración de las actividades turísticas en un solo sector puede llegar a desgastar tanto a los sitios (recursos o atractivos) como a la misma comunidad que vive el día a día con los movimientos que genera el turismo 
en su entorno. Es por eso que se propone de alguna manera la descentralización de la oferta congregada en la comuna 13; se hace referencia al graffitour como manifestación cultural y artística, la cual se puede llevar a otros espacios de la ciudad, en los que a manera de lienzo, las calles y paredes sean igualmente intervenidas por los colectivos culturales que laboran en el barrio San Javier y sus alrededores, en medio de un proceso de apertura cultural que beneficie a la ciudad misma, sus habitantes y los turistas que llegan hasta esta.

La margen occidental de Medellín presenta una concentración importante de espacios deportivos de primer nivel. Las unidades deportivas como la Atanasio Girardot, Andrés Escobar, María Luisa Calle, René Higuita y Mariana Pajón, entre otras -en su mayoría referencian a celebres deportistas de Medellín, que a lo largo de los años han marcado huella en el deporte mundial-, generan una nueva opción para quienes llegan a la ciudad a disfrutar de su tiempo libre de manera saludable.

Esta concentración se presta para construir un vínculo directo con la gran cantidad de disciplinas deportivas que se practican en estos espacios adecuados y mejorados de acuerdo con las necesidades del momento. El deporte, como estilo de vida para los habitantes de Medellín y como oferta complementaria para quienes visitan la ciudad, es la propuesta de articulación de estos elementos que se conectan fácilmente a través del sistema de bicicletas públicas del Valle de Aburrá EnCicla. Se busca ampliar el número de actividades deportivas que se pueden llevar a cabo en los diferentes espacios en los que se promueve el deporte como actividad primordial para el bienestar de las personas. Un elemento que facilita esta lógica propuesta es la gratuidad de muchas de estas ofertas. El INDER (Instituto de Deporte y Recreación de Medellín), principal institución encargada de la promoción del deporte, es la encargada de administrar los espacios e impartir la formación deportiva, lo que abre aún más el espectro de posibles beneficios para quienes quieran y puedan hacer uso eficiente de estas unidades deportivas y demás espacios adecuados para tal fin.

\section{Conclusiones}

Muchos de los turistas que llegan a la ciudad de Medellín buscan experiencias enriquecidas por el patrimonio, gastronomía, eventos culturales, deportivos, calidades ambientales, destrezas sociales y de conocimiento, que a su vez deben ser articuladas con una buena movilidad facilitada por una red de transportes, y otros servicios complementarios, como hoteles, seguridad, señalización, puntos de información, entre otros. Para el caso de Medellín, «laboratorio turístico en construcción», debe ser motivo para trabajar en el desarrollo económico, social y cultural, con sostenibilidad. En los últimos años han desarrollado de manera incipiente ejercicios puntuales de vinculación y asociación de recursos o atractivos con posibles servicios, empoderando a la comunidad de una manera incluyente y logrando una transformación positiva de la ciudad.

Este laboratorio turístico caso Medellín permite ir más allá de los conceptos tradicionales, teniendo una mirada asociativa, complementaria y agrupando por unidades espaciales turísticas, que permite potencializar la oferta no solo en turismo de reuniones y convenciones, científico y médico, sino en función de la cultura y la naturaleza, vinculando las actividades humanas al territorio en la generación de valor.

La ciudad está identificando nuevas ofertas de recorridos y productos turísticos asociados a procesos de transformación territorial con énfasis en lo cultural, productivo, social, técnico, ambiental, tecnológico y comercial, que motivan a atraer nuevas corrientes de viajeros y a aumentar los tiempos de estadía en la ciudad, que propician mayores derrames económicos y generación de nuevos empleos. La ciudad deberá realizar esfuerzos en la formalidad, legalidad y estándares de servicios de sus prestadores, propiciar trabajos colaborativos y sinérgicos entre los actores y realizar ejercicios investigativos de orden académico, para generar nuevas miradas y prospectivas del devenir turístico local en su sostenibilidad, competitividad e inclusión social.

La institucionalidad pública y privada no propiamente turística deberá realizar un esfuerzo de comprensión y participación efectiva y vinculante con la estructura de gobernanza del turismo local. Los ejercicios de doble esfuerzo y en algunos casos en contravía de las necesidades que tiene la ciudad para su consolidación como destino turístico es la resultante de dicha descoordinación institucional, sumado a esto el hecho de no asimilar la realidad del crecimiento de la actividad y de su rol local en lo económico y social desde los actores propiamente turísticos, que en algunos casos 
dudan de lo que está sucediendo en el último lustro. Una ciudad, que tiene un flujo actual de visitantes y turistas internacionales relativamente cercano a un destino tradicionalmente consolidado y reconocido a nivel nacional, debe despertar el interés de propios y extraños.

De otro lado están los actores no turísticos, que son escépticos y no le prestan atención al fenómeno que sucede, porque lo consideran temporal o sin fundamentación científica para su sostenibilidad; eso se ve reflejado en las conversaciones, documentos técnicos e incluso en la definición de políticas públicas de la ciudad en los aspectos social y económico de mediano y largo plazo.

Otro elemento que es importante referir es la mirada de ciudad-región, de la cual se dice, se verbaliza, pero en la práctica cuesta trabajo la articulación, la inclusión y concertación de acciones. Se siente que la ciudad y el resto del territorio departamental no lograrán llegar a acuerdos en ejercicios de planeación, promoción, difusión, diseño de productos, complementariedad en servicios, transferencia de buenas prácticas, ampliación de capacidades de negociación y generación de sinergias para trabajar conjuntamente con otros entes de características nacionales o internacionales. Es un reto para los próximos años, si deseamos que la actividad del turismo incida en los territorios como estrategia de desarrollo local, de inclusión social, sostenibilidad y competitividad territorial.

\section{Referencias bibliográficas}

Alcaldía de Medellín. (2016). Plan de Desarrollo Medellín Cuenta Con Vos 2016-2019. Medellín

Borthiry, N. (2002). Gestión pública municipal del turismo: Un desafío basado en el fortalecimiento institucional y en la participación. Mar de Plata, Argentina: Universidad Nacional del Mar del Plata: Facultad de ciencias económicas.

Boullon, Robert. (1985). Planificación del Espacio Turístico (Primera edición ed.). México, D.F.: Trillas S.A de C.V.

Marchena Gómez, M., y Vera Rebollo, J. F. (1997). Análisis Territorial del Turismo. Andalucía: Ariel.

Ministerio de Comercio, Industria y Turismo. (2010). Metodología para la elaboración del inventario de atractivos turísticos. Bogotá D.C.

Ministerio de Comercio, Industria y Turismo. (2016). Informe de flujos de visitantes a nivel nacional por departamentos y ciudades. Bogotá D.C.

Presidencia de la República. (2014). Plan sectorial de turismo 2014-2018. Bogotá, D.C.

Procolombia. (2016). Recuperado de http://www.procolombia.co/actualidad-internacional/vacacional/conozca-el-potencial-del-turismo-urbano-y-sus-tendencias

Viceministerio de turismo y FONTUR. (2012). Plan de Desarrollo Turístico de Antioquia 2012-2015. Medellín, Colombia. 